\title{
Reflets
}

Revue ontaroise d'intervention sociale et communautaire

\section{Équipes communautaires de traitement intensif : description et expérience des équipes parrainées par l'Hôpital Montfort}

\section{Carl Brouillette, Bernard L’abbé et Manon Tremblay}

Volume 8, numéro 1, printemps 2002

La réadaptation : son visage français en Ontario

URI : https://id.erudit.org/iderudit/026377ar

DOI : https://doi.org/10.7202/026377ar

Aller au sommaire du numéro

Éditeur(s)

Reflets : Revue ontaroise d'intervention sociale et communautaire

ISSN

1203-4576 (imprimé)

1712-8498 (numérique)

Découvrir la revue

Citer cet article

Brouillette, C., L’abbé, B. \& Tremblay, M. (2002). Équipes communautaires de traitement intensif : description et expérience des équipes parrainées par l'Hôpital Montfort. Reflets, 8(1), 114-124. https://doi.org/10.7202/026377ar

Tous droits réservés (C Reflets : Revue ontaroise d'intervention sociale et communautaire, 2002
Ce document est protégé par la loi sur le droit d'auteur. L'utilisation des services d'Érudit (y compris la reproduction) est assujettie à sa politique d'utilisation que vous pouvez consulter en ligne.

https://apropos.erudit.org/fr/usagers/politique-dutilisation/ 


\section{Équipes communautaires de traitement intensif : description et expérience des équipes parrainées par 1'Hôpital Montfort.}

Carl Brouillette

Équipes communautaires de traitement intensif, Hôpital Montfort

\section{Bernard L'abbé}

Programme de santé mentale, Hôpital Montfort

\section{Manon Tremblay}

Centre national de formation en santé, École des sciences de la réadaptation (ergothérapie), Université d'Ottawa

\section{Introduction}

Les services de santé mentale de l'Ontario font actuellement l'objet d'une transformation destinée à " augmenter la capacité du système de santé à offrir un éventail complet et intégré de services de traitement, de réadaptation et de soutien mettant l'accent sur les services communautaires" (MSSLD 1998b:3). L'implantation de cette réforme recommande que les services développés respectent les normes stipulées dans le document approuvé par le Ministère et intitulé : Examen des meilleures pratiques de la réforme des soins de 
la santé mentale (Unité de recherche sur les systèmes de santé, Institut psychiatrique Clarke 1997).

Concernant le plan de mise en œuvre du MSSLD, les écrits affirment que le système de santé mentale réformé doit inclure dans un continuum complet et coordonné de services, des équipes communautaires de traitement intensif (ÉCTI), mieux connues sous le vocable anglophone Assertive Community Treatment (ACT). Ainsi, l'Hôpital Montfort a reçu le mandat de gérer entièrement une équipe unilingue francophone sur le territoire d'Ottawa en plus de co-gérer avec l'Hôpital psychiatrique de Brockville/ Hôpital Royal d'Ottawa, une équipe desservant les Comtés Unis de Prescott-Russell (milieu rural).

ÉCTI est un modèle de services développé par Leonard Stein, Mary Ann Test et leurs collègues dans les années 1970 à Madison, Wisconsin. Mentionnons que ce modèle est également connu sous d'autres noms anglophones et francophones. Parmi les plus fréquents en français, nous retrouvons : équipe communautaire de traitement intensif, équipe communautaire de traitement actif, équipe de traitement dynamique et équipe de suivi intensif dans la communauté.

Le modèle ÉCTI ne s'est pas implanté aussi rapidement que ses auteurs l'auraient espéré.Toutefois, un sondage réalisé en 1996 aux États-Unis a recensé un total de 396 équipes dans 34 états (Meisler 1997). D'ici 2002, les services ÉCTI seront présents dans plus 50 états américains (Flynn 1998).

\section{Le développement des ÉCTI en Ontario}

Plusieurs facteurs politiques, économiques et socioculturels ont influencé le développement de ces équipes à l'extérieur des ÉtatsUnis. Au Canada, le document Examen des meilleures pratiques de la réforme des soins de la santé mentale (1997) est un document charnière sur lequel s'est appuyé le gouvernement de l'Ontario pour développer l'implantation de 51 ÉCTI dans la province. 
Afin d'assurer une rigueur et une uniformité des équipes ÉCTI, le gouvernement a publié deux documents complémentaires: Franchir les étapes: Cadre de prestation des services de santé mentale et des services de soutiens connexes (MSSLD 1998a) et Standards for Assertive Community Treatment Teams (MSSLD 1998c). Ces deux documents dressent les lignes directrices à suivre pour la création de cette nouvelle approche de traitement. Le gouvernement de l'Ontario tient également une conférence annuelle entièrement consacrée aux ÉCTI.

Dans la réforme des services en Ontario, le ministère a adopté un cadre conceptuel pour clarifier les rôles et les responsabilités des fournisseurs de services. Les ÉCTI sont définies comme suit :

des services de santé mentale hautement spécialisés offerts dans la communauté... aux personnes ayant une maladie mentale grave qui présentent des troubles complexes, rares et instables. Ces services de traitement, de réadaptation et de soutien sont intégrés dans chaque type de programme ou de service et leur prestation est confiée à une équipe multidisciplinaire (MSSLD 1998a).

Enfin, le ministère a adopté des mesures pour favoriser une pratique basée sur des faits scientifiques. Parmi les outils de mesures de résultats exigés par le ministère, nous retrouvons le Psychosocial Rehabilitation Toolkit (PSR Toolkit) traduit en français comme l'Ensemble d'outils de mesures en réadaptation psychosociale.

\section{Caractéristiques générales du modèle équipe communautaire de traitement intensif}

Un programme ÉCTI consiste en une équipe clinique autonome composée de divers professionnels de la santé qui offrent des services spécialisés sur une base intensive aux personnes aux prises avec un trouble mental grave. Les programmes ÉCTI ont une 
approche holistique ou globale. L'équipe intervient donc dans de multiples aspects du fonctionnement de la personne. Elle peut intervenir aussi bien sur le pland de la prise de médicaments que sur celui des volets résidentiel, professionnel et financier (Bond, Drake, Mueser, \& Latimer 2001).

Quoique le modèle ait évolué depuis sa création, les experts s'entendent pour dire que les éléments suivants sont essentiels : équipe multidisciplinaire, intégration des services (traitement, réadaptation et soutien), approche d'équipe, nombre de clients par intervenant limité, contacts dans la communauté, gestion des médicaments (évaluation, choix et dosage approprié), emphase sur les activités de la vie quotidienne, accès rapide aux services (services disponibles $24 \mathrm{~h} /$ jour), repérage actif, services individualisés, durée illimitée des services, assistance professionnelle et psychoéducation/soutien aux familles (McGrew \& Bond 1995; MSSLD 1998c).

\section{Clientèle cible}

Les services sont destinés aux personnes aux prises avec un trouble mental grave (TMG). Un TMG est défini selon trois critères : diagnostic, incapacité et durée (Schinnar, Rothbard, Kanter, et al. 1990). La majorité des personnes aux prises avec un TMG ont un diagnostic de schizophrénie, de trouble schizo-affectif ou de trouble bipolaire. Néanmoins, certaines personnes ont d'autres diagnostics psychiatriques tels que dépression majeure et trouble d'anxiété sévère. Le deuxième critère est établi à partir des incapacités sur le plan du fonctionnement occupationnel, par exemple, les soins personnels, la productivité (emploi, bénévolat), les loisirs et les relations sociales. Finalement, pour répondre au critère de durée, la personne doit avoir reçu des services de traitement pendant un certain temps, ce qui peut inclure de multiples hospitalisations psychiatriques, une hospitalisation d'une durée de plusieurs mois ou la participation à un programme intensif de traitement. 
Ces critères viennent préciser les éléments requis pour dire qu'une personne est aux prises avec un trouble mental grave. Même si la personne répond à tous ces critères, cela ne signifie pas qu'elle est automatiquement admissible aux services d'une ÉCTI.En effet, des critères d'admission additionnels ont été établis par le ministère (MSSLD 1998c) pour que les ÉCTI ciblent mieux les clients. Dans la région d'Ottawa, le comité d'évaluation des demandes a retenu six de ces critères : la personne doit être âgée de 16 ans et plus; elle doit avoir un diagnostic primaire autre que celui d'abus de drogues ou d'alcool (la priorité est accordée aux clients avec un diagnostic de psychose); la personne doit démontrer une forte utilisation des services de santé (c'est-à-dire au moins 50 jours d'hospitalisation [cumulés] au cours de la dernière année, et/ou incapacité d'accéder à des services pour cause d'itinérance, d'incarcération et ce, pour une période de plus de 60 jours); elle doit avoir des difficultés significatives de fonctionnement telles qu'une incapacité à maintenir les activités de la vie quotidienne, une incapacité à assurer sa sécurité et une incapacité à participer aux services existants; les cas complexes sont également jugés prioritaires (coexistence de problème d'abus de substance pour plus de 6 mois, troubles psychiatriques concomitants, problème avec le système judiciaire); les clients avec un trouble sévère de la personnalité antisociale et qui représentent un danger pour le personnel de l'ÉCTI ne sont pas admis (Central Intake 2000).

\section{Services offerts}

Globalement, le traitement vise à évaluer, soulager, réduire ou gérer les manifestations du trouble. Les services dispensés par une ÉCTI sont classés selon trois grandes catégories de fonctions des services. Il s'agit du traitement, de la réadaptation et du soutien.

Les services de traitement comprennent: l'identification et l'évaluation des signes et symptômes, l'évaluation diagnostique, les évaluations bio-psychosociales, l'administration des médicaments et le suivi de la médication, le traitement de l'abus de 
substance, le counseling et la psychothérapie (individu et famille), l'hospitalisation et l'intervention en situation de crise. Bien que les ÉCTI visent à diminuer les hospitalisations, il n'en demeure pas moins que l'hôpital constitue un lieu de traitement privilégié dans certaines circonstances et constitue un des éléments du continuum de services.

La réadaptation se décrit comme un processus de développement des habiletés et/ou de soutiens environnementaux requis pour surmonter les obstacles au fonctionnement dans la communauté (logement, emploi, éducation, socialisation, activités de la vie quotidienne). La réadaptation se caractérise par des activités destinées à maintenir, améliorer ou rétablir la capacité d'une personne à être en santé et retrouver un équilibre occupationnel : apprentissage des activités de la vie quotidienne, formation à la vie en société, aux relations interpersonnelles et aux activités de loisirs. La réadaptation psychiatrique comprend aussi des interventions dont les objectifs sont la gestion des symptômes, l'acquisition des habiletés nécessaires pour répondre aux demandes environnementales, la modification des caractéristiques de l'environnement de même que le renforcement et le développement d'un réseau de soutien social.

Pour leur part, les services de soutien aident la personne à maintenir une bonne qualité de vie et à accéder aux différents services (sociaux, de santé et communautaires). Le suivi et la coordination des services, les initiatives d'entraide et d'enrichissement et les services de soutien social (halte-accueil, programmes de récréation, programmes d'éducation, interventions de bénévoles) (MSSLD 1998b) sont caractéristiques des services de soutien.

\section{ÉCTI d'Ottawa}

Dans toutes les équipes ÉCTI, les membres, peu importe leur discipline, doivent partager de façon équitable une variété d'activités qui soutiennent le client dans la communauté. Il peut 
s'agir par exemple, de téléphoner à un client pour l'encourager à réaliser ses activités de la vie quotidienne, d'évaluer des situations de crise (ex. suicide) et développer un plan de gestion de crise, d'assurer la livraison et la supervision des médicaments ou de rencontrer un client pour lui offrir du soutien dans divers domaines de sa vie.

L'expertise de chaque discipline est déterminante pour l'équipe et dans la prestation des services à la clientèle. En effet, pour répondre aux besoins spécifiques des usagers, les différents membres doivent mettre à profit leur apport spécifique. Notre équipe est actuellement composée à temps plein d'une adjointe de programme, d'un ergothérapeute, d'un gestionnaire d'équipe, de deux infirmières, d'un intervenant en réadaptation et toxicomanie, d'une psychiatre et d'une travailleuse sociale. L'ergothérapeute est bien préparé au niveau de l'approche de réadaptation psychiatrique et de la réadaptation professionnelle. C'est pourquoi il a comme responsabilité de faciliter les démarches de réinsertion professionnelle :soutien à l'emploi (supported employment) et soutien à l'éducation (supported education). Il procède également à l'évaluation des besoins professionnels de la clientèle et assure au besoin la coordination avec d'autres services, (par ex. le Programme ontarien de soutien aux personnes handicapées-programme de soutien à l'emploi).

Comme notre gestionnaire d'équipe possède une expérience et des connaissances appropriées auprès des personnes présentant un trouble de personnalité limite, il est la personne ressource pour ces cas. L'intervenant en réadaptation et toxicomanie possède une longue expérience en matière d'alcoolisme et de toxicomanie. En préconisant une approche intégrée, il accompagne et soutient les clients ayant un diagnostic concomitant d'alcoolisme dans leur implication auprès des Alcooliques anonymes (réunions, explication des 12 étapes AA ).

Les services sont dispensés de $8 \mathrm{~h} 30$ à $21 \mathrm{~h} 00$ du lundi au vendredi. En dehors de ces heures, les deux ÉCTI ont un intervenant disponible pour répondre aux situations d'urgence. Autrement dit, la clientèle a accès à un intervenant 24 heures par jour et ce, 365 jours par année. 
Dans un souci de garantir aux clients l'accès à des services qui répondent à leurs besoins, l'ÉCTI d'Ottawa a développé des liens pertinents et appropriés avec d'autres services offerts au sein de la région d'Ottawa.

L'accueil de toutes les équipes de la ville d'Ottawa est centralisé. Le bureau d'accueil est situé dans des locaux partagés avec l'Association canadienne de la santé mentale. Ce service évalue toutes les demandes de services et les dirige vers l'équipe la plus appropriée.

Sur une base trimestrielle, l'ÉCTI participe à des comités, notamment le Comité aviseur régional, the Eastern Ontario ACT Network et the Technical Advisory Panel. Ces comités ont pour mandat de maintenir entre autres des services de qualité, répondant aux normes gouvernementales.

Quoique l'ÉCTI offre des services de soutien continus et d'une durée illimitée à sa clientèle, elle demeure soucieuse de favoriser le développement de services intégrés, accessibles et pertinents au développement des clients. Ainsi, l'ÉCTI a un représentant au sein d'un nouveau partenariat d'organismes francophones sur le territoire de la ville d'Ottawa dont le rôle est de :

Favoriser la consolidation et le développement de services diversifiés par l'entremise d'un partenariat entre les agences du secteur des services de santé mentale et les autres secteurs francophones (loisirs, éducation, travail, logement,...) afin d'assurer une meilleure intégration et participation sociale des personnes francophones aux prises avec un problème de santé mentale. (Partenariat d'organismes francophones pour le développement de services de nature récréationnelle et professionnelle (Janvier 2002). Document inédit, Ottawa.)

Les membres de l'ÉCTI collaborent aussi à d'autres projets et partenariats : la clinique de trouble de personnalité limite de l'Hôpital Montfort, le projet pour les sans-abri dont le mandat est de développer des services et des logements pour ces personnes, le regroupement des spécialistes à l'emploi. 
Finalement, l'ÉCTI entretient des liens étroits avec le système de soutien du client et les unités d'hospitalisation. En effet, lors d'hospitalisation, un intervenant assiste aux réunions cliniques de l'unité de soins en vue de préparer le retour à la communauté.

\section{Efficacité des ÉCTI et résultats préliminaires en Ontario}

De tous les modèles de suivi communautaire (case management), le modèle ÉCTI est celui pour lequel il y a eu le plus de recherches évaluatives. Bond et al. (2001), en accord avec Bedell, Cohen \& Sullivan (2000), concluent que ÉCTI diminue les hospitalisations psychiatriques tout en augmentant la stabilité résidentielle. Cette approche de traitement aiderait également à contrôler de façon modérée les symptômes et à améliorer la qualité de vie subjective. De surcroit, elle engendre un plus grand engagement des clients face à leur traitement.

Dans la plus récente analyse sur le coût-efficacité des ÉCTI, Latimer (1999) démontre que les ÉCTI, comparés à des services traditionnels de suivi communautaire en santé mentale, ont un meilleur coût-efficacité pour les personnes ayant un vécu marqué de services hospitaliers.

Dans le cadre de la réforme en Ontario, des mesures précises ont été adoptées afin d'établir des attentes en matière de rendement et des normes applicables aux programmes ÉCTI. Une collecte de données auprès des programmes est actuellement en cours pour documenter les résultats de ceux-ci et établir la contribution de ces équipes à l'amélioration du système de santé. Les résultats de cette recherche devraient être connus au cours de la présente année. 


\section{Conclusion}

Les programmes ÉCTI font partie intégrante de la réforme de santé mentale en Ontario. La réforme vise à répondre le plus adéquatement possible aux besoins des personnes aux prises avec un trouble mental grave. Les programmes ÉCTI sont largement reconnus. Leur valeur est bien démontrée aux États-Unis et est soutenue par les faits scientifiques. Les équipes parrainées par l'Hôpital Montfort sont récentes, mais leur implantation servira à démontrer le bien-fondé de ce type d'intervention.

\section{Références}

BEDELL, J.R., COHEN, N.L., \& SULLIVAN, A. (2000). " Case management : the current best practices and the next generation of innovation ", Community Mental Health Journal, 36, 179194.

BOND, G., DRAKE, R.E., MUESER, K.T., \& LATIMER, E. (2001). « Assertive Community Treatment for People with Severe Mental Illness : Critical Ingredients and Impact on Patients ", Dis Manage Health Outcomes, 9:3, 141-159.

CENTRAL INTAKE (2000). Critères d'admission. Document inédit. Ottawa

FLYNN, L.M. (1998). Commentary. Schizophrenia Bulletin, 24, 30-32.

LATIMER, E. (1999). "Economic impacts of assertive community treatment : a review of the literature ", Canadian Journal of Psychiatry, 44, 443-454.

MCGREW, J.H., \& BOND, G. (1995). «Critical ingredients of assertive community treatment : judgements of the experts ", Journal of Mental Health Administration, 22, 113-125.

MEISLER, N. (1997). "Assertive community treatment initiatives : results from a survey of selected state mental health authorities ", Community Support Network News, 11:4, 3-5.

MINISTÈRE DE LA SANTÉ ET DES SOINS DE LONGUE DURÉE [MSSLD] (1998a). Franchir les étapes : Cadre de prestation des services de santé mentale et des services de soutien connexes.

MINISTÈRE DE LA SANTÉ ET DES SOINS DE LONGUE DURÉE [MSSLD] (1998b). Franchir les étapes: Plan de mise en cuvre continue de la réforme du système de santé mentale.

MINISTĖRE DE LA SANTÉ ET DES SOINS DE LONGUE DURÉE [MSSLD] (1998b). Standards for Assertive Community Treatment Teams.

PARTENARIAT D'ORGANISMES FRANCOPHONES POUR LE DÉVELOPPEMENT DE SERVICES DE NATURE RÉCRÉATIONNELLE ET PROFESSIONNELLE (Janvier 2002). Document inédit. Ottawa.

SCHINNAR,A., ROTHBARD, A., KANTER, R. et al. (1990). "An empirical literature review of definitions of severe and persistent mental illness ", American Journal of Psychiatry, 147, 16021608 . 
UNITÉ DE RECHERCHE SUR LES SYSTÈMES DE SANTÉ, Institut psychiatrique Clarke (1997). Examen des meilleures pratiques de la réforme des soins de la santé mentale : examen de la documentation. Ottawa On, Publications Santé Canada. 